\title{
Nanoscale Cancer Therapeutics: Advances, Hurdles and Hopes
}

Moffatt $S^{*}$

Faculty of Engineering, Computing and Allied Sciences, Regent University College of Science and Technology, Ghana.

\section{Introduction}

Cancer constitutes many heterogeneous diseases [1] having varied genetic and epigenetic abnormalities [2, 3] afflicting millions of people globally. Despite substantial progress in elucidating the complex molecular mechanisms underlying its cause [4], improvements in terms of therapeutic index have largely remained unchanged, while concomitant increases in the death rates have been steadily climbing over the past five decades [5, 6]. Failure to deliver effective treatment to cancer patients in the past has been the inability to get enough of the drug in the right amounts and form, and to the right place.

The traditional drug delivery methods, which basically include the Oral and IV routes of administration, are still the most widely used today, yet each has its disadvantages. Oral delivery via tablets or capsules is largely inefficient due to exposure of the pharmaceutical agent to the metabolic processes of the body. Therefore, a larger dose is often required while not compromising the maximum effectiveness of the drug. Traditional IV administration is even much more problematic. Specificity for IV injectable drugs is often low, necessitating large amounts of a drug be injected into a patient, creating a high concentration of the drug in the blood stream that could potentially lead to toxic side effects. The potential of eliminating a tumorous outgrowth without any collateral damage through nanomaterial-based drug delivery has created significant interest and nanoparticles form the basis for bio nanomaterials [7] and major efforts in designing drug delivery systems are based on functionalized nanoparticles [8, 9]. As postulated by Paul Ehrlich in 1906, an ideal therapeutic agent, is one that works like a "magic bullet", by reaching exclusively, its target [10].

This concept has been advanced by encapsulating drugs into various nanoscale delivery systems, in order to enhance its target specificity. The main idea is to make drug fate dependent on the carrier in which it is encapsulated. Throughout various fields of science and technology, a push towards the use of nanoscale technology for medical interventions is well underway. The use of nanotechnology (particles between 10 nanometers and 1000 nanometers in size) in medical applications (i.e. nanomedicine) has the potential to produce low-cost, self-replicating systems that could revolutionize the scientific landscape [7].

Earlier work with micro-carriers, soon gave way to nanocarriers because these could be administered either locally (eye, lungs, nose, etc.) or systemically (intravenously). Further, their small size enhances intracellular diffusion, which is a big boost for molecular medicine. For more than 40 years, research into the therapeutic abilities of drug-loaded nanocarriers has become a burgeoning science [11-13], resulting in the term nanomedicine, i.e. biodegradable and/or biocompatible submicron-sized colloidal particles encapsulating a drug. Recent advances into physical and biochemistry have resulted in nanomedicines of various architectures and surface properties [14-16].

This review first presents the various types of foundational materials for nanomedicines, followed by the physiological barriers encountered, and finally, how these barriers can be overcome. Alternative routes of administration, new trends in biotherapeutics, nanomedicine and nanotheranostics are also discussed.

\section{Different Types of Nanomedicines}

The materials used to synthesize nanomedicines range from organic molecules to inorganic ones. Liposomes are the very first nanomedicines and consist of vesicles in an aqueous suspension, with each vesicle comprising a bilayer composed of natural or synthetic phospholipids (Figure 1). Their size can be adjusted by extrusion or sonication. Hydrophilic drugs such as doxorubicin [17] or 5-fluorouracil [18] can be encapsulated in the internal core, while hydrophobic or amphiphilic drugs such as amphotericin B [19] are inserted within the bilayer. The type of phospholipid matrix can affect the encapsulation efficacy and the fate of the liposomes after systemic administration. The upscaling of lipo-

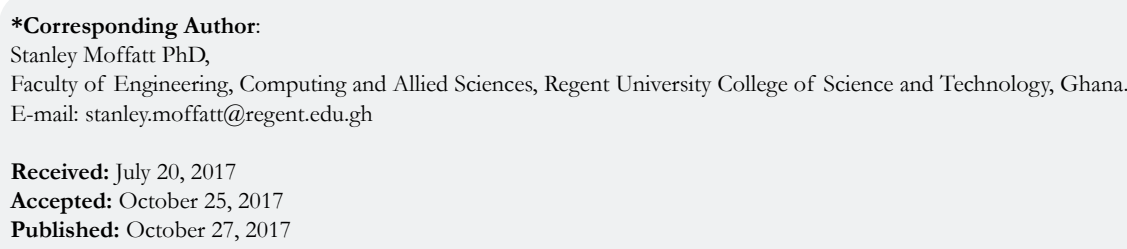


Figure 1. Schematic representation of lipid nanomedicines (top) or polymers (bottom).

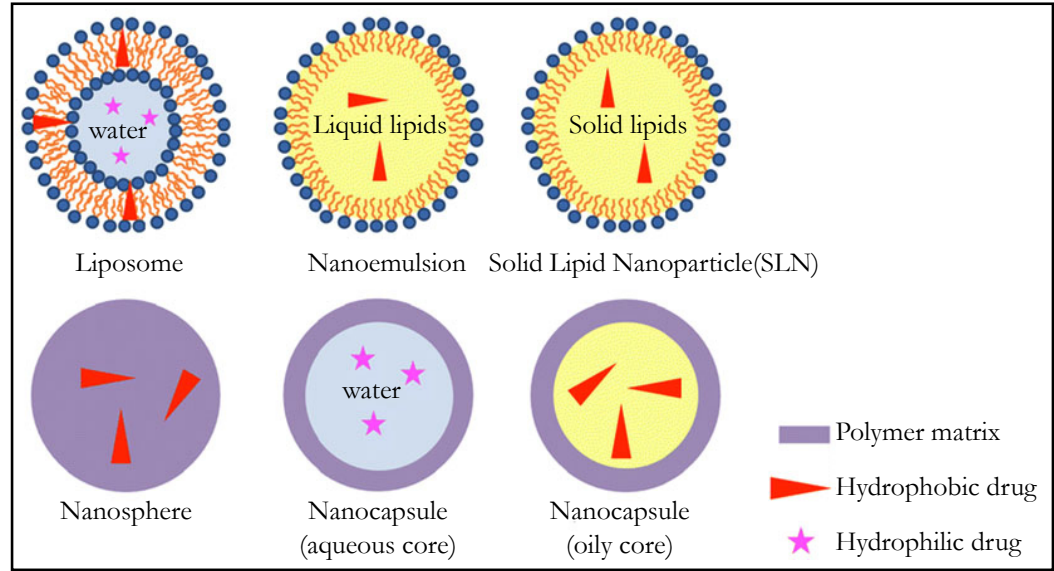

somal formulations may be limited drug leakage, non-specific targeting, the physical stability of the dispersions, and clearance by the mononuclear phagocytic system (MPS) [20].

Liposomes are small artificial and spherical vesicles that have at least one lipid bilayer. It usually has an aqueous solution core surrounded by a hydrophobic membrane, in the form of a lipid bilayer. A liposome can hence be loaded with hydrophobic and/or hydrophilic molecules. By preparing liposomes in a solution of DNA or drugs (which cannot readily diffuse through the membrane) they can be (indiscriminately) but homogeneously delivered past the lipid bilayer [21]. To deliver the molecules to a site of action, the lipid bilayer can fuse with other bilayers such as the cell membrane, in order to deliver the liposome contents. Their size, hydrophobicity and hydrophilicity of liposome systems render them very promising for drug delivery. Liposomes are generally classified based on their size, surface charge, lipid composition, and the method of preparation. Further more, the bilayer composition determines the charge and fluidity [22-24].

Lipid nanoemulsions are lipid-based nanosystems used for drug encapsulation. They consist of layers of lipid droplets surrounded by a layer of surfactant (Figure 1) which can encapsulate hydrophobic soluble drugs in the lipid core. They can also entrap amphiphilic molecules [25] or adsorb macromolecules through electrostatic interactions [26]. Apart from the significant energy involved in their production, one other major disadvantage of nanoemulsions is their poor stability after systemic administration. High levels of surfactants used to stabilize nanoemulsions have been reported to have serious deleterious effects [27].

Solid lipid nanoparticles (SLNs) are another class of lipid-based nanoparticles, which bear the advantages of physical stability, protection from degradation, tolerability, and controlled release. SLNs have a solid lipid core (usually triglycerides or complex glyceride mixtures) which are stabilized by a layer of surfactant (Figure 1). Majority of hydrophobic SLN-containing drugs are prepared from solvent emulsification-evaporation methods [28]. Most of the lipids which are cationic in nature have been used in formulating iRNA, DNA, and other oligonucleotides to yield nanomedicines [29]. Because cationic lipids can condensate nucleic acids to form nano-objects due to their electrostatic interactions, they protect nucleic acids from degradation, thereby enhancing their entry into cells to release their payloads. Polymer nanoparti- cles have been the second most used materials for nanomedicine formulations. Their biodegradability properties made them even more suitable for human applications. Increasing research point to greater use of nanoparticles than liposomes due to their better stability, allowing for the successful encapsulation of many drugs. Consequently, many different polymers such as poly (lactide-coglycolide), poly-dl-lactide, polysaccharides, and polyesters, have been synthesized and formulated for nanomedicines applications.

These polymers can also be formulated into nanospheres (matrix systems) or nanocapsules (vesicular systems) depending on the method of preparation. (Figure. 1). The preparation methods developed so far have been classified into two main categories; via a polymerization reaction or directly from a preformed polymer. Nanoparticle preparation generally consist of introducing a monomer into the dispersed phase of an emulsion, or dissolving the monomer in a non-solvent of the polymer. Polymerization reactions then occur in a nucleation step followed by a growth step.

Two protocols have been presented for preparing nanoparticles from preformed artificial polymers. The first protocol is emulsification of non water-miscible organic solutions of preformed polymers into an aqueous phase containing surfactants, followed by removal of solvents under reduced pressure. This method, named solvent emulsion-evaporation has already been applied to poly-dl-lactide [30]. This process can also be modified to yield capsules with a perfluorcarbon core within a polymer shell. This is then mixed in the solvent together with the polymer. To encapsulate hydrophilic drugs, a double emulsion (water-in-oil-inwater) is formed with the internal aqueous phase (Figure 1). The second protocol is called nanoprecipitation, and is based on the precipitation of a solvent polymer after adding a non-solvent of the polymer. The solvent and also the non-solvent of the polymer should be reciprocally miscible. The progressive addition of the polymer solution to the non-solvent typically ends up in the formation of nanospheres, generally not greater than two hundred $\mathrm{nm}$ in size. Polymeric micelles have additionally been proposed, stemming from the self-assembly of amphiphilic copolymers, resulting in spherical aggregates of a few nanometers in diameter. Since hydrophobic moieties sort out as a core, by encapsulating hydrophobic drugs they can modify their solubility and therefore their biodistribution. Amphiphilic copolymers can also form a bilayer that encloses an internal aqueous compartment called polymersomes [31]. 
One limitation of classical nanomedicines is their low drug-loading limit. Analysts have beaten this downside by orchestrating prodrug particles that can self-collect into nanomedicines. One such strategy squalenoylation, which is a concept involving the formation of chemical linkage between a drug and squalenic acid, a primer of cholesterol synthesis, followed by nano assembly of the new molecules via nanoprecipitation [33]. Molecules which have been successfully coupled to squalene, for example paclitaxel [34], siRNA [35] and nucleosides [36], have produced nanomedicines that have increased drug loading to around $50 \%$ of the bearer weight. Studies indicate a superior efficacy of the prodrug nano assemblies as compared with the free drug in tumor therapy [37].

Materials utilized are not constrained to natural mixes and several groups have optimized inorganic nanomaterials to permit proficient encapsulation. Albeit, metal organic frameworks (MOFs) were originally developed for hydrogen storage, it has been demonstrated that these can be modified to yield nanosized MOFs that epitomize medications such as azidothymidine (AZT) or busulfan with high drug loading capacities [38].

\section{Crossing Physiological Barriers}

One promising aspect of nanomedicine is that nano-pharmaceuticals have greater bioavailability. This property translates into the body absorbing the drug compound easier, faster and utilize it more effectively. Further, routes of administration, such as intravenous, oral, transdermal, and pulmonary can be made easier. This is a huge improvement over many currently available drugs with poor bioavailability. As per their surface properties, they can be grouped into three generations. As non-self particles after intravenous injection, nanomedicines are equally subjected to several physiological barriers within the body. Their clearance may occur even before they arrive at the targeted diseased site. Understanding and successfully navigating the physiological barriers is therefore paramount in order to predict the fate of injected nanomedicines and their biodistribution profiles.

Nanomedicines are typically prepared as colloidal suspensions or buffer solution, stability and fate of which can be assessed after intravenous administration. In the presence of salts, proteins and enzymes in the vascular compartment, nanomedicines can undergo hydrolysis, cleavage or aggregation. Nanomedicines below ten nanometers are filtered from the blood stream via the glomerular capillaries of the kidneys. In the case of nanomedicines up to five hundred nanometers, and depending on their surface properties, opsonisation could occur, followed by macrophage take-up and sequestration in the liver, spleen and bone marrow. Their preferred accumulation in macrophages was exploited to treat intracellular infections by delivering antibiotics to infected macrophages [39].

Strategies to reduce opsonisation led to the second generation of nanomedicines which is characterized by passive tumor-targeting. To reduce opsonisation, one can consider surface modification with either natural or synthetic polymers (Figure 2). Reducing opsonisation means the reduction of protein adsorption to the nanomedcines, and this increases the circulation time in the blood compartment, thereby increasing the half-life in the tumors as a result of the enhanced permeation and retention (EPR) effect. Smaller nanomedicines extravasate more efficiently through neovessels. This feature of PEGylated nanomedicines has led to the has resulted in the production and use of $\operatorname{Doxil}^{\circledR} / \mathrm{Caelyx}^{\circledR}$ in the clinic. It consists of doxorubicin-encapsulated PEGylated liposomes, for the treatment of cancer. Liposomal doxorubicin formulations reduce cardiac toxicity more efficiently than free doxorubicin. Indeed, clinical studies with equivalent doses PEGylated liposomal doxorubicin or free drug increased the doxorubicin levels in the tumor by about 16-fold when compared to the free drug [18].

The strategy of passive targeting of tumors has not been attractive since nanomedicines reaching their targets is usually below $10 \%$ of the injected dose. Furthermore, since plasma protein adsorption deceases with PEGylation, uptake by the RES is also decreased. It has also been observed in the clinic that the EPR effect is only functional in certain patients [25]. Finally, research in animals suggest that intravenous injections of the liposomes could trigger an immune response leading to rapid clearance of the PEGylated liposomes after a second injection [43]. Attention has recently been shifted towards several biopolymers such as

Figure 2. Schematic representation of the plain, PEGylated and targeted liposomes.

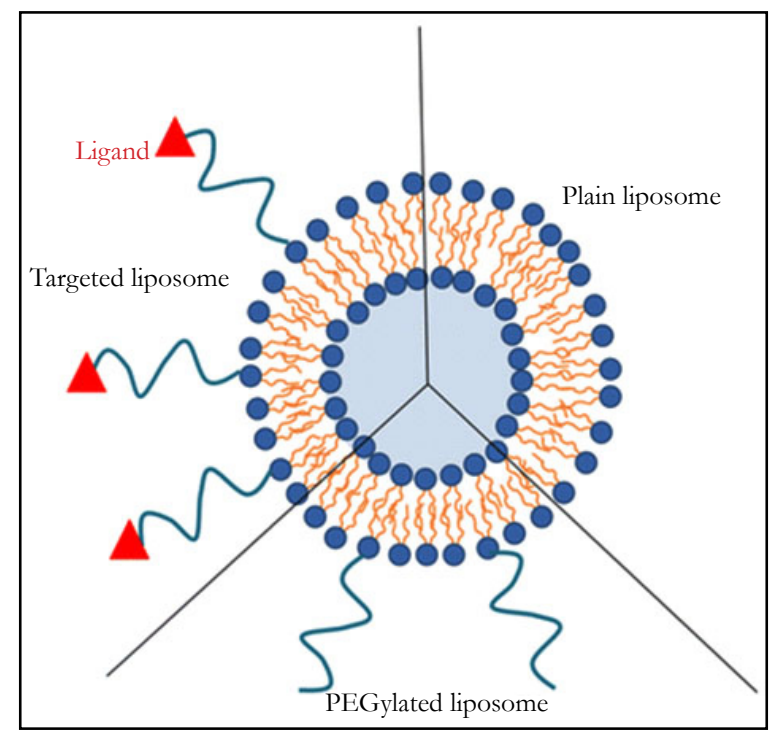


polysaccharides to replace PEG in the formulations [44].

The third generation of nanomedicines relies on active targeting. Active targeting has so far been the most effective by way of applying external magnetic field to increase local concentration. Several authors explored this strategy by placing the superparamagnetic iron oxide (SPIO) over subcutaneously implanted tumors, leading to enhanced tumor-specific accumulation. This targeting strategy has however been difficult to translate to the clinic due to the skills need in implanting a magnet near the tumor site.

Alternatively, there is the chemical approach by coupling the nanomedicine surface with targeting ligands such as antibodies [45], peptides [46], proteins [47], aptamers [48] or small molecules such as folic acid [49], that recognize receptors on the tumor site. (Figure 2). Active targeting reduces significantly non-specific binding compared to ligand-receptor interactions. Usually a spacer is needed between the ligand and the nanoparticle. Consequently, the best site is to attach the ligand at the extremity of the polymer coating to avoid opsonisation.

Active targeting was based on the hypothesis that biomarkers like integrins are over expressed on neovessels or epithelial cells, and that the expression differential between targeted and non-targeted tissues could be as high as ten. Active targeting hence allows greater uptake of nanomedicines due to enhanced receptor-mediated endocytosis. Nevertheless, because the biomarker expression varies with the stage of the disease, the expected response to targeted nanomedicines could be affected. There is a school of thought that is proposing that exclusive receptors for malignant cells should also be considered. A typical example is Glypican (GPC), which is absent in normal tissue but over-expressed in hepatocellular carcinoma. A proof of principle study by Park et al. [33], demonstrated the specific uptake by Hep G2 cancer cells using anti-GPC3 antibody-encapsulated PEGylated nanoparticles.

\section{Beyond the Intravenous Route}

Apart from the classical intravenous administration, other routes of administration for nanomedicines such, as the oral, pulmonary, and the ocular route are under consideration. The oral route, however, seems to be the most preferred choice by the pharmaceutical industry, perhaps due to the ease of application. Even though nanomedicines can protect the degradation of labile drugs (like insulin) by the acidic gastric $\mathrm{pH}$, many of the successfully developed nanomedicines encapsulating insulin for oral delivery are yet to reach the market, presumably because of the narrow therapeutic window. The idea of extending the residence time in the intestine in order to favour drug absorption has resulted in the use of bioadhesive polymers such as chitosan or thiolated chitosan on the nanoparticle surface [51].

In the area of pulmonary delivery, either locally or systemic, nanomedicines have also been quite successful. Pulmonary delivery of targeted nanomedicines at the vascularized alveoli, is a better alternative than the parenteral route since it allows the administration of poorly absorbed molecules. The bioavailability of nanoparticles after lung administration via liquid nebulization or dry powders encapsulated into Trojan particles has seen some success [52].
Nanomedicines have also been quite efficacious in ocular delivery were developed to extend the residence time of the drug at the surface of the eye. In particular, cationic nanoemulsions have been developed for the treatment of the dry eye syndrome. Intraocular injections of nanomedicines have also been considered for the posterior segment of the eye, but has not been very successful due to the difficult in reaching its target by either topical administration or intravenous administration.

\section{Nanotheranostics}

One interesting system that has been developed is the attachment of a contrast agent to the nanomedicine cohort to allow for imaging, diagnosis and treatment i.e. nanotheranostic system. The term theranostic is derived from the words therapy and diagnostics. Nanotheranostic systems are therefore designed to image the biodistribution of nanomedicines, diagnose, determine the extent of disease spread, and also monitor its action and efficacy [52]. In nanotheranostic systems, there should be an optimal delivery of two payloads: the bioactive molecule (peptide, nucleic acid, chemotherapeutic drug etc.), and the imaging probe (quantum dots, fluorophores, etc.).

The most commonly used imaging modality has been Magnetic Resonance Imaging (MRI) because of its excellent resolution and its ability to permeate several organs including the brain. Further, several MRI contrast agents (Gd chelates, super paramagnetic iron oxides (SPIOs), ultrasmall super paramagnetic iron oxides (USPIOs), as well as perfluorocarbons (Figure 3) [53], have been developed and customized for easy co-encapsulation into nanomedicines. Analysis of the release of doxorubicin from liposomes, co-encapsulated with manganese as the MRI contrast agent has been extensively done [54], by comparing the measurements from HPLC and histology, a method that has gained popularity for effective imaging and evaluation of chemotherapeutic protocols [55].

Optical imaging has been considered a visualization modality option for soft tissues in nanotheranostics, but their application is limited due to the scattering properties in the visible region of the light spectrum (less than $700 \mathrm{~nm}$ ). The scattering however decreases in the near-infrared region (700-900 nm), for optical imaging, commonly called the "biological window". The NIR fluorescence dye Cy5.5 has been used to label paclitaxel-loaded chitosan-based nanomedicines [55]. In vivo, NIR fluorescence in vivo clearly delineated the tumor and signal intensity correlated with nanomedicine concentration. Optical imaging also clearly depicted the nanomedicine biodistribution and allowed the monitoring of the tumor growth rate in response to treatment (Figure 4) [55]. Even though optical NIR probes besides organic dyes, such as quantum dots, can also be loaded into nanomedicines, it is important that the observed tissues be located close to the radiation source since the depth of NIR light penetration in tissue is less than $1 \mathrm{~cm}$. Currently, clinical use of optical imaging remains limited.

\section{Conclusion}

Nanomedicines have evolved as the next generation of therapeutics due to the recurring issues with poor bioavailability, poor 
Figure 3. 19F MR image superimposed with $1 \mathrm{H}$ MR image of a longitudinal cross section of a mouse $7 \mathrm{~h}$ after intravenous injection of PLGA-PEG nanocapsules of perlfluorooctyl bromide for a $720 \mathrm{~mm}^{3}$ tumor (white dotted circles). Color images adapted from Diou et al., [53].

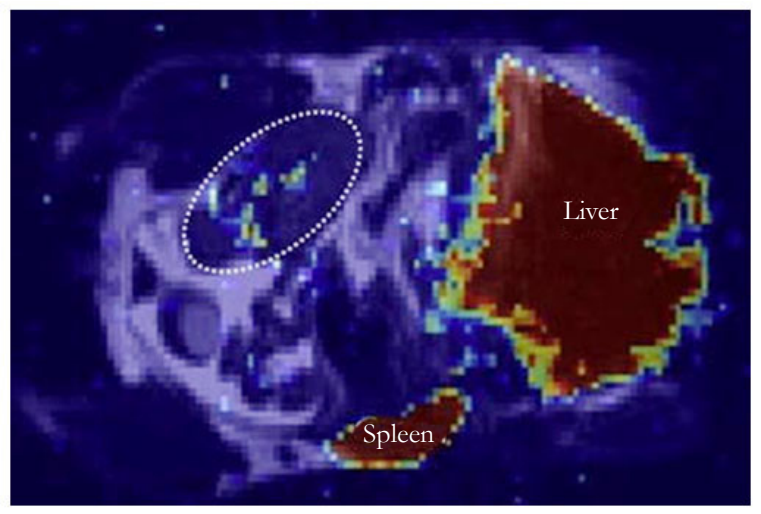

Figure 4a. In vivo imaging of tumor-bearing mice using Cy5.5-labeled chitosan-based nanoparticles (CNPs). Figure. 4b $\mathrm{H} \& \mathrm{E}$ staining confirmed positive pectoral staining. Fluorescence microscopy also showed CD-31-positive angiogenic vessels (yellow) and DAPI-stained tumor cells (blue). Cy5.5-labeled CNPs injected intravenously were visualized in tumor tissues (red). Figure. 4c Kinetics of tumor targeting with free Cy5.5, Cy5.5-labeled polymers, and Cy5.5-labeled CNPs. Fig. 4d Tumor-to-background (muscle) ratio as a function of time after administration of free or labelled Cy5.5. All data represent mean \pm SE. Color images adapted from Kim et al., [55].

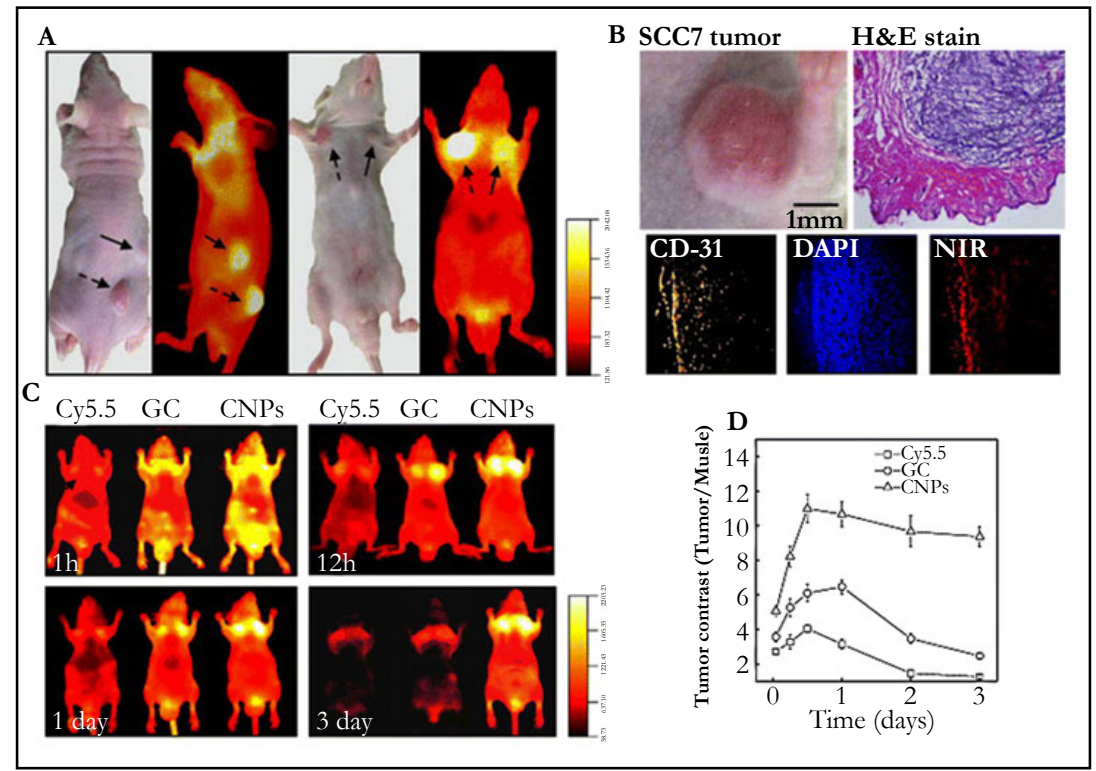

stability, drug toxicity, and harmful side effects of the traditional means of drug administration. Nanomedicines are derived from organic and inorganic materials and their physical and chemical properties can be adjusted for specific applications. As a result of advanced research, nanomedicines have been tuned for oral, ocular or intravenous delivery. The new concept of nanotheranostics seem interesting with great potential as a therapeutic modality, but remain to be fully explored for clinical use.

Quite a number of studies have been carried out on the toxicity of nanoparticles, but there is currently no acceptable protocol for safety assessment of nanomaterials. A clear regulatory framework to address the potential health impacts, within the wider context of monitoring, evaluating and assessment, must be an integral part of efforts towards nanotechnology innovation. Commercially, nanotechnology is expected to contribute over five trillion dollars to GDP by the 2025. Therefore, rigorous research, that will attract commercial investment in nanotechnology, should provide an overall societal benefit. Overall, nanomedicines will have to be upgraded from preclinical research to clinical application if the toxicity issues are better predicted and the scale-up and engineering of these complex structures prove profitable.

\section{References}

[1]. Marte B. Tumour heterogeneity. Nature. 2013 Sep 19;501(7467):328-37.

[2]. Kircher M, Witten DM, Jain P, O'Roak BJ, Cooper GM, Shendure J. A general framework for estimating the relative pathogenicity of human genetic variants. Nat Genet. 2014 Mar;45(3):310-5. Epub 2014 Feb 2. PubMed PMID: 24487276

[3]. Suvà ML, Riggi N, Bernstein BE. Epigenetic reprogramming in cancer. Science. 2013 Mar 29;339(6127):1567-1570. PubMed PMID: 23539597.

[4]. Seyfried TN, Flores RE, Poff AM, D’Agostino DP. Cancer as a metabolic disease: implications for novel therapeutics. Carcinogenesis. 2014 Mar;35(3):515-27. Epub 2013 Dec 16. PubMed PMID: 24343361.

[5]. Sliwkowski MX, Mellman I. Antibody therapeutics in cancer. Science. 2013 Sep 13;341(6151):1192-1198. PubMed PMID: 24031011.

[6]. Service RF. Materials and biology. Nanotechnology takes aim at cancer. Science. 2005 Nov;310(5751):1132-1134. PubMed PMID: 16293748. 
[7]. Wang L, Zhao W, Tan W. Bioconjugated silica nanoparticles: development and applications. Nano Res. 2008 Aug;1(2):99-115.

[8]. Yokoyama M, Miyauchi M, Yamada N, Okano T, Sakurai Y, Kataoka K, Inoue S. Polymer micelles as novel drug carrier: adriamycin-conjugated poly(ethylene glycol)-poly(aspartic acid) block copolymer. J Control Rel. 1990 Jan;11(1-3):269278.

[9]. Yokoyama M, Okano T, Sakurai Y, Ekimoto H, Shibazaki C, Kataoka K. Toxicity and antitumor activity against solid tumors of micelle-forming polymeric anticancer drug and its extremely long circulation in blood. Cancer Res. 1991 Jun;51(12):3229-3236. PubMed PMID: 2039998.

[10]. Ehrlich P: Referatüber die Genese des Carcinoms. Verh Dtsch Pathol Ges. 1908;12:13-32.

[11]. Ahmed RZ, Patil G, Zaheer Z. Nanosponges - a completely new nano-horizon: pharmaceutical applications and recent advances. DrugDevInd Pharm. 2013 Sep;39(9):1263-1272. PubMed PMID: 22681585.

[12]. Laurance J. Scientists develop nanoparticle method to help tackle major diseases. The Independent. 2012 Nov 18

[13]. Getts DR, Martin AJ, McCarthy DP, Terry RL, Hunter ZN, Yap WT, et al. Micro particles bearing encephalitogenic peptides induce T-cell tolerance and ameliorate experimental autoimmune encephalomyelitis. Nat Biotechnol. 2012 Dec;30(12):1217-1224. PubMed PMID: 23159881.

[14]. Hollmer M. Carbon nanoparticles charge up old cancer treatment to powerful effect. Fierce drug delivery. 2012 Feb 17.

[15]. Garde D. Chemo bomb' nanotechnology effective in halting tumors. Fierce drug delivery. 2012 Apr 25

[16]. Peiris PM, Bauer L, Toy R, Tran E, Pansky J, Doolittle E, et al. Enhanced delivery of chemotherapy to tumors using a multicomponent nanochain with radio-frequency-tunable drug release. ACS Nano. 2012 May 22; 6(5): 4157 4168. PubMed PMID: 22486623.

[17]. Gabizon A, Catane R, Uziely B, Kaufman B, Safra T, Cohen R, et al. Prolonged circulation time and enhanced accumulation in malignant exudates of doxorubicin encapsulated in polyethylene-glycol coated liposomes. Cancer Res. 1994 Feb 15;54(4):987-992. PubMed PMID: 8313389.

[18]. Joondeph BC, Peyman GA, Khoobehi B, Yue BY. Liposome-encapsulated 5-fluorouracil in the treatment of proliferative vitreoretinopathy. Ophthalmic Surg. 1988 Apr;19(4):252-256. PubMed PMID: 3362494.

[19]. Lopezberestein G, Mehta R, Hopfer RL, Mills K, Kasi L, Mehta K, et al. Treatment and prophylaxis of disseminated infection due to Candida albicans in mice with liposome-encapsulated amphotericin-B. J Infect Dis. 1983 May;147(5):939-945.PubMed PMID:6842027.

[20]. Uster PS. Liposomes as drug carriers: trends and progress. Gregoriadis G, editor. Wiley: Chichester; 1988.

[21]. Barenholz Y, Cevc G. Structure and properties of membranes. In: Dekker M, editor. Physical chemistry of biological surfaces. New York: Basel; 2000. $171-241$

[22]. Sahoo SK, Labhasetwar V. Nanotech approaches to drug delivery and imaging. Drug Discovery Today. 2003 Dec 15;8(24):1112-20. PubMed PMID: 14678737

[23]. Gabizon A, Goren D, Cohen R, Barenholz Y. Development of liposomal anthracyclines: from basics to clinical applications. J Control Release. 1998 Apr 30;53(1-3):275-279. PubMed PMID: 9741935. doi: 10.1016/S01683659(97)00261-7.

[24]. Allen TM. Liposomes. Opportunities in drug delivery. Drugs. 1997;54 Suppl 4:8-14. PubMed PMID: 9361956.

[25]. Spagnul A, Bouvier-Capely C, Phan G, Rebiere F, Fattal E. Calixarene-entrapped nanoemulsion for uranium extraction from contaminated solutions. J Pharm Sci. 2010 Mar;99(3):1375-1383. PubMed PMID: 19780139.

[26]. Bruxel F, Cojean S, Bochot A, Teixeira H, Bories C, Loiseau PM, et al. Cationic nanoemulsion as a delivery system for oligonucleotides targeting malarial topoisomerase II. Int J Pharmaceut. 2011 Sep 20;416(2):402-409. PubMed PMID: 21291974

[27]. Gutierrez JM, Gonzalez C, Maestro A, Sole I, Pey CM, Nolla J. Nano-emulsions: new applications and optimization of their preparation. Curr Opin Colloid Interface Sci. 2008 Aug;13(4):245-251.

[28]. Cortesi R, Esposito E, Luca G, Nastruzzi C. Production of lipospheres as carriers for bioactive compounds. Biomaterials. 2002 Jun;23(11):22832294. PubMed PMID: 12013175.

[29]. Fattal E, Couvreur P, Dubernet C. Smart delivery of antisense oligonucleotides by anionic pH-sensitive liposomes. Adv Drug Deliv Rev. 2004 Apr 23;56(7):931-946. PubMed PMID: 15066753.

[30]. Fattal E, Rojas J, Roblottreupel L, Andremont A, Couvreur P. Ampicillinloaded liposomes and nanoparticles: comparison of drug loading, drug release and in vitro antimicrobial activity. J Microencapsul. 1991 Mar; 8(1):29-36. PubMed PMID: 1880689.

[31]. Vanderhoff JW, El-Aasser MS, Ugelstad J, inventors; Polymer emulsification process. United States Patent US 4177177 A. 1979 Dec 4.

[32]. Lee JS, Feijen J. Polymersomes for drug delivery: design, formation and characterization. J Control Release. 2012 Jul 20;161(2):473-483. PubMed PMID: 22020381

[33]. Desmaele D, Gref R, Couvreur P. Squalenoylation: a generic platform for nanoparticular drug delivery. J Control Release. 2012 Jul 20;161(2):609-618. PubMed PMID: 21840355.

[34]. Caron J, Maksimenko A, Wack S, Lepeltier E, Bourgaux C, Morvan E, et al. Improving the antitumor activity of squalenoyl-paclitaxel conjugate nanoassemblies by manipulating the linker between paclitaxel and squalene. Adv Healthc Mater. 2013 Jan; 2(1):172-185. PubMed PMID: 23213041.

[35]. Raouane M, Desmaele D, Gilbert-Sirieix M, Gueutin C, Zouhiri F, Bourgaux C, et al. Synthesis, characterization, and in vivo delivery of siRNAsqualene nanoparticles targeting fusion oncogene in papillary thyroid carcinoma. J Med Chem. 2011 Jun 23;54(12):4067-4076. PubMed PMID: 2151161

[36]. Hillaireau H, Dereuddre-Bosquet N, Skanji R, Bekkara-Aounallah F, Caron J, Lepetre S, et al. Anti-HIV efficacy and biodistribution of nucleoside reverse transcriptase inhibitors delivered as squalenoylated prodrug nanoassemblies. Biomaterials. 2013 Jul;34(20):4831-4838. PubMed PMID: 23562054.

[37]. Reddy LH, Renoir JM, Marsaud V, Lepetre-Mouelhi S, Desmaele D, Couvreur P. Anticancer efficacy of squalenoyl gemcitabine nanomedicine on 60 human tumor cell panel and on experimental tumor. Mol Pharm. 2009 Oct;6(5):1526-1535. PubMed PMID: 19634915

[38]. Horcajada P, Chalati T, Serre C, Gillet B, Sebrie C, Baati T, et al. Porous metal-organic-framework nanoscale carriers as a potential platform for drug delivery and imaging. Nat Mater. 2010 Feb;9(2):172-178. PubMed PMID: 20010827.

[39]. Semiramoth N, Di Meo C, Zouhiri F, Said-Hassane F, Valetti S, Gorges R, et al. Self-assembled squalenoylated penicillin bioconjugates: an original approach for the treatment of intracellular infections. ACS Nano. 2012 May 22;6(5):3820-3831. PubMed PMID: 22482704.

[40]. Abraham SA, Waterhouse DN, Mayer LD, Cullis PR, Madden TD, Bally MB. The liposomal formulation of doxorubicin. Methods Enzymol 2005;391:71-97. PubMed PMID: 15721375

[41]. Diou O, Tsapis N, Giraudeau C, Valette J, Gueutin C, Bourasset F, et al. Long-circulating perfluorooctyl bromide nanocapsules for tumor imaging by (FMRI)-F-19. Biomaterials. 2012 Aug;33(22):5593-5602. PubMed PMID: 22575831.

[42]. Crommelin DJA, Florence AT. Towards more effective advanced drug delivery systems. Int J Pharm. 2013 Sep 15;454(1):496-511. PubMed PMID: 23415662.

[43]. Ishida T, Ichihara M, Wang X, Yamamoto K, Kimura J, Majima E. Injection of PEGylated liposomes in rats elicits PEG-specific IgM, which is responsible for rapid elimination of a second dose of PEGylated liposomes. J Control Release. 2006 May 1;112(1):15-25. PubMed PMID: 16515818.

[44]. Bertholon I, Vauthier C, Labarre D. Complement activation by core-shell poly(isobutylcyanoacrylate)-polysaccharide nanoparticles: influences of surface morphology, length, and type of polysaccharide. Pharm Res. 2006 Jun;23(6):1313-1323. PubMed PMID: 16715369.

[45]. Koshkaryev A, Sawant R, Deshpande M, Torchilin V. Immunoconjugates and long circulating systems: origins, current state of the art and future directions. Adv Drug Deliv Rev. 2013 Jan; 65(1):24-35. PubMed PMID: 22964425.

[46]. Kumar M, Yigit M, Dai G, Moore A, Medarova Z. Image-guided breast tumor therapy using a small interfering RNA nanodrug. Cancer Res. 2010 Oct 1;70(19):7553-7561. PubMed PMID: 20702603

[47]. Bartlett DW, Su H, Hildebrandt IJ, Weber WA, Davis ME. Impact of tumor-specific targeting on the biodistribution and efficacy of siRNA nanoparticles measured by multimodality in vivo imaging. Proc Natl AcadSci USA. 104(39):15549-15554. PubMed PMID: 17875985.

[48]. Wang AZ, Bagalkot V, Vasilliou CC, Gu F, Alexis F, Zhang L, et al. Superparamagnetic iron oxide nanoparticle-aptamer bioconjugates for combined prostate cancer imaging and therapy. ChemMedChem. 2008 Sep;3(9):1311-1315. PubMed PMID: 18613203.

[49]. Yang X, Grailer JJ, Rowland IJ, Javadi A, Hurley SA, Steeber DA, et al. Multifunctional SPIO/DOX-loaded wormlike polymer vesicles for cancer therapy and MR imaging. Biomaterials. 2010 Decx;31(34):9065-9073. PubMed PMID: 20828811.

[50]. Park JO, Stephen Z, Sun C, Veiseh O, Kievit FM, Fang C, et al. Glypican-3 targeting of liver cancer cells using multifunctional nanoparticles. Mol Imaging. 2011 Feb;10(1):69-77. PubMed PMID: 21303616

[51]. Reineke J, Cho DY, Dingle YL, Cheifetz P, Laulicht B, Lavin D, et al. Can bioadhesive nanoparticles allow for more effective particle uptake from the small intestine? J Control Release. 2013 Sep 28;170(3):477-484. PubMed PMID: 23796432

[52]. Sung JC, Padilla DJ, Garcia-Contreras L, Verberkmoes JL, Durbin D, Peloquin CA, et al. Formulation and pharmacokinetics of self-assembled rifampicin nanoparticle systems for pulmonary delivery. Pharm Res. 2009 
Aug;26(8):1847-1855. PubMed PMID: 19407933.

[53]. deSmet M, Heijman E, Langereis S, Hijnen NM, Grüll H. Magnetic resonance imaging of high intensity focused ultrasound mediated drug delivery from temperature-sensitive liposomes: an in vivo proof-of-concept study. J Control Release. 2011 Feb 28;150(1):102-110. PubMed PMID: 21059375.

[54]. Viglianti BL, Ponce AM, Michelich CR, Yu D, Abraham SA, Sanders L, et al. Chemodosimetry of in vivo tumor liposomal drug concentration using MRI. Magn Reson Med. 2006 Nov;56(5):1011-1018. Pubmed PMID: 17029236.

[55]. Kim K, Kim JH, Park H, Kim YS, Park K, Nam H, et al. Tumor-homing multifunctional nanoparticles for cancer theragnosis: simultaneous diagnosis, drug delivery, and therapeutic monitoring. J Control Release. 2010 Sep 1;146(2):219-227. PubMed PMID: 20403397.
Special Issue on

"Nanomedicine and Biotherapeutics"

Edited by:

Stanley Saamoah Moffatt, Nova Southeastern University, USA.

E-mail: stanley.moffatt@regent.edu.gh

Viorel Simion, Laboratory of Nucleic acids transfer by non-viral vectors, CNRS Orleans, France. viorel.simion@cnrs-orleans.fr 